\title{
Combined effects of semiconductor gain dynamics, spin dynamics and thermal shift in polarization selection in VCSELs
}

\author{
M. San Miguel ${ }^{* a}$, S. Balle ${ }^{a}$, J. Mulet ${ }^{a}$, C.R. Mirasso ${ }^{b}$, E. Tolkachova ${ }^{c}$, and J.R. Tredicce ${ }^{c}$ \\ ${ }^{a}$ Instituto Mediterraneo de Estudios Avanzados, IMEDEA, (CSIC-UIB) \\ E-07071 Palma de Mallorca, Spain. \\ ${ }^{b}$ Department de Física, Universitat de les Illes Balears, E-07071 Palma de Mallorca, Spain. \\ ${ }^{c}$ Institut Non Lineaire de Nice, CNRS UMR 6618, 06560 Valbone, France.
}

\begin{abstract}
We discuss mechanisms of polarization switching (PS) in Vertical Cavity Surface Emitting Lasers (VCSELs) within a mesoscopic approach based on an explicit form of a frequency-dependent complex susceptibility of the QW semiconductor material. Cavity anisotropies, spin carrier dynamics and thermal shift of the gain curve are also taken into account in this framework. For large birefringence we find a PS due to thermal shift. For small birefringence we find a different PS, from the high-gain to the low-gain polarization state, that occurs at constant temperature. We characterize polarization partition noise in terms of power spectra. Transverse effects for PS in gain guided VCSELs are also considered.
\end{abstract}

Keywords: VCSEL, Polarization Switch, Semiconductor susceptibility, RIN

\section{INTRODUCTION}

Vertical Cavity Surface Emitting Lasers (VCSELs) typically emit linearly polarized (LP) light and, for bias currents relatively close to threshold, in the fundamental transverse mode. Often, the LP state selected at threshold becomes unstable as the injection current is increased, and a switch to the orthogonal LP state occurs for some value of the injection current. ${ }^{1}$ Understanding the physical mechanisms that determine polarization selection and polarization switching (PS) is a first step towards the development of applications based on polarization control such as optical switching, information processing or storage, etc.

Polarization selection has been often attributed to linear cavity anisotropies, being birefringence one of the dominant. $^{2}$ A mesoscopic description of polarization, carrier spin and nonlinear semiconductor gain dynamics has been put forward through a Spin Flip Model. ${ }^{3}$ In this context it has been shown that nonlinear anisotropies which result from the semiconductor dynamics are also important mechanisms of polarization selection. In particular PS has been seen to be caused by phase-amplitude dynamics that follow from the combined effect of birefringence and saturable dispersion associated with the linewidth enhancement factor, $\alpha .^{4,5}$ A limitation of the original formulation of this explanation is that the model does not incorporate a frequency dependent gain, therefore it does not take into account either nonlinear gain differences between the LP states, or the effects of thermal shift of the cavity resonances and the gain peak. Gain differences between the two LP states and thermal shift have been, however, invoked as a dominant mechanism of $\mathrm{PS}^{6}$ : Gain differences appear because of the different frequency of the LP states in a birefringent optical cavity, and a change of sign in the gain difference between the two LP states is caused by the thermal shift of the gain curve when increasing the injection current. A frequency dependent gain was already introduced within the context of the SFM model in ref. [7] giving a good description of general properties of polarization switching and transverse mode competition. However, the complex susceptibility in ref. [7] is based on a simple two-level approach and the choice of the $\alpha$-factor is justified as an effective description of thermal lensing effects.

In this paper we elaborate an extension of the SFM which incorporates a realistic spectral dependence of the gain and index of refraction of active semiconductor quantum well $(\mathrm{QW})$ structures. $^{8}$ Within this framework there is

*Correspondence: Email: maxi@imedea.uib.es; WWW: http://www.imedea.uib.es/Photonics; Telephone: 34-971 173229; Fax: 34-971 173426

In Physics and Simulation of Optoelectronic Devices VIII, R.F. Binder, P. Blood, M. Osinski Editors, Proceedings of SPIE Vol. 3944 (2000) 
room for the different mechanisms of PS described above. We find thermally induced PS, but for low birefringence and finite values of the carrier spin flip rate we also find PS from the high-gain to the low-gain polarization states caused by coupled phase-amplitude nonlinear dynamics.

The outline of the paper is as follows. In Section 2 we recall the main features of the complex frequency-dependent quantum well susceptibility which enters as a main ingredient of our dynamical model. Section 3 describes our results for the different mechanisms of PS in the fundamental transverse mode. We also discuss the changes in the Relative Intensity Noise spectrum through the PS. Finally Section 4 contains some first results on transverse effects and transverse beam profile.

\section{QUANTUM WELL ELECTRONIC SUSCEPTIBILITY}

We describe the optical field in terms of the slowly varying amplitudes of its circularly polarized components $E_{ \pm}$. Each component interacts only with a part, $N_{ \pm}$, of the total carrier density $N=N_{+}+N_{-}$. The carrier densities $N_{ \pm}$are associated with carrier populations of different spin and tend to be equalized by spin flip processes at a rate $\gamma_{j}{ }^{1,3}$ A scheme of the interactions and band structure considered is shown in Fig. 1.

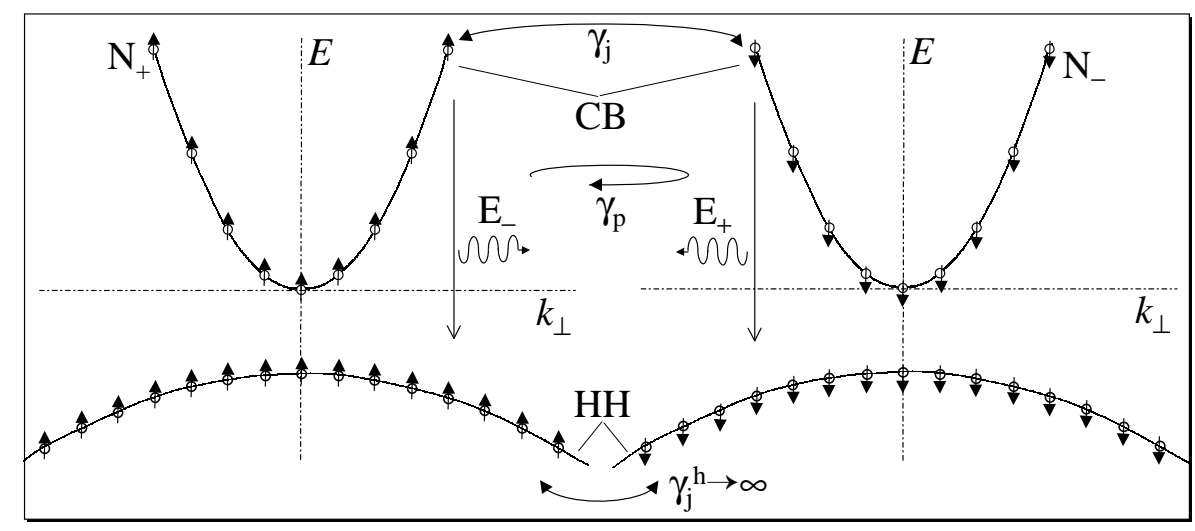

Figure 1. An extension of the SFM based on a four level system. We consider a conduction band (CB) and only one heavy-hole $(\mathrm{HH})$ spin sub-bands. The spin of the electrons are equalized at rate $\gamma_{j}$ and instantaneously for the holes. $^{9}$ The circularly polarized components of the field $E_{ \pm}$fields are coupled by birefringence $\gamma_{p}$.

The interaction of each circularly polarized component of the field with its corresponding carrier density is described by a frequency-dependent complex susceptibility $\chi_{ \pm}$which incorporates the basic nonlinear properties of the QW gain and index of refraction. ${ }^{10}$ The form of the susceptibility described below has been shown to give a good description, in particular, of semiconductor optical amplifiers. ${ }^{11}$ The calculation of the susceptibility $\chi_{ \pm}$involves a summation over all $k$-states in the first Brillouin zone weighted with the carrier occupancy of each state. It can be analytically evaluated when the following simplifying assumptions are made ${ }^{10}:$ i) the dipole matrix element between the valence and conduction bands is independent of $k$, ii) the width of each transition is also independent of $k$, iii) all carriers are in quasi-equilibrium within each band, and iv) low temperature limit. When only one electron and one hole (parabolic) bands are considered it is found that,

$$
\chi\left(\omega_{ \pm}, D_{ \pm}\right)=\chi_{0}\left[\ln \left(1-\frac{2 D_{ \pm}}{u_{ \pm}+i}\right)+\ln \left(1-\frac{D_{+}+D_{-}}{u_{ \pm}+i}\right)-\ln \left(1-\frac{b}{u_{ \pm}+i}\right)\right],
$$

where $D_{ \pm} \equiv N_{ \pm} / N_{t}$ is the carrier density per spin orientation normalized to the (total) transparency carrier density, $N_{t}$. The dependence on the frequency of the fields arises through

$$
u_{ \pm}=\frac{\omega_{ \pm}}{\gamma_{\perp}}+\Delta+\sigma\left(D_{+}+D_{-}\right)^{1 / 3},
$$

where $\Delta=\left(\Omega-\omega_{t}\right) / \gamma_{\perp}$ is the detuning between the cavity resonance $\Omega$ and the nominal transition frequency normalized to the linewidth, $\gamma_{\perp}$, of the optical transitions for fixed $k$. This linewidth corresponds to the inverse 
of the dephasing time of the material polarization. $\Omega+\omega_{ \pm}$are the optical frequencies of each circularly polarized wave. Band-gap renormalization effects have been included in (1) through $\sigma$ which describes the band-gap shrinkage with carrier density. The parameter $b$ is linked to the total energy span of the conduction and valence bands and it determines the optical response of the QW material in the absence of carriers, i.e., it sets the background index of refraction and absorption of the unpumped active region. Finally, $\chi_{0}$ is the device-dependent parameter that determines the material gain.

From a microscopic point of view one defines lattice and electron plasma temperatures. Evolution equation for these magnitudes can indeed be obtained and integrated with the rest of electric and carrier equations. ${ }^{12}$ In the mesoscopic approach that we follow here only an averaged temperature is taken into account. In particular, as the main part of generation and recombination events take place in the active region of the device, we are interested in the active region temperature. Within this context, a primary thermal effect, namely, the relative shift of the cavity frequency and the material gain spectrum, is easily taken into account in (1) by letting $\Delta$ to depend on temperature. Since the gain spectrum redshifts 4-5 times faster than the cavity resonance, increasing temperature corresponds to increasing $\Delta$. We consider only temperature changes small enough to neglect thermal changes in material gain and transparency carrier density.

A subtle technical point is how to make use of the susceptibility (1) (given in the frequency domain) in nonlinear dynamical equations written as differential equations in time. What is needed is an analytical expression for the material polarization $\tilde{\mathcal{P}}_{ \pm}(w)$ as function of time. We can start from the constitutive relation, in frequency domain, $\tilde{\mathcal{P}}_{ \pm}\left(w_{ \pm}\right)=\varepsilon_{0} \chi\left(w_{ \pm}\right) \tilde{\mathcal{E}}_{ \pm}\left(w_{ \pm}\right)$. Our fields have a narrow interval of frequencies compared with the gain broad band, so that we can use a Slowly Varying Approximation (SVA). We take $\Omega$ as the carrier frequency, $\omega_{ \pm}$as the SVA frequency, and $w_{ \pm}=\Omega+\omega_{ \pm}$

$$
\begin{aligned}
& \mathcal{E}_{ \pm}(t)=E_{ \pm}(t) e^{-i \Omega t} \\
& \mathcal{P}_{ \pm}(t)=P_{ \pm}(t) e^{-i \Omega t}
\end{aligned}
$$

By Fourier transform of (3), (4) we obtain the constitutive relation for the SVA components:

$$
\tilde{P}_{ \pm}\left(\omega_{ \pm}\right)=\varepsilon_{0} \chi\left(\Omega+\omega_{ \pm}\right) \tilde{E}_{ \pm}\left(\omega_{ \pm}\right)
$$

so that

$$
P_{ \pm}(t)=\int_{-\infty}^{\infty} \frac{d \omega_{ \pm}}{2 \pi} \varepsilon_{0} \chi\left(\Omega+\omega_{ \pm}\right) \tilde{E}_{ \pm}\left(\omega_{ \pm}\right) e^{-i \omega_{ \pm} t}
$$

Since $\omega_{ \pm} \ll \Omega$ we can expand $\chi\left(\Omega+\omega_{ \pm}\right)$in a power series and integrate the series term by term. The carrier densities are assumed to be constant in the material polarization time scales $\left(\gamma_{\perp}^{-1}\right)$.

$$
\begin{aligned}
P_{ \pm}(t) & =\varepsilon_{0} \int_{-\infty}^{\infty} \frac{d \omega_{ \pm}}{2 \pi}\left[\chi(\Omega)+\omega_{ \pm} \chi^{\prime}(\Omega)+\ldots\right] \tilde{E}_{ \pm}(\omega) e^{-i \omega_{ \pm} t}= \\
& =\varepsilon_{0}\left[\chi(\Omega) E_{ \pm}(t)+\chi^{\prime}(\Omega) i \dot{E}_{ \pm}(t)+\ldots\right] \approx \varepsilon_{0} \chi\left(\Omega+i \frac{\dot{E}_{ \pm}(t)}{E_{ \pm}(t)}\right) E_{ \pm}(t)
\end{aligned}
$$

where we have defined $\left.\chi^{\prime}(\Omega) \equiv \frac{\partial \chi(\Omega+\omega \pm, D)}{\partial \omega}\right|_{\omega_{ \pm}=0}$. Equation (7) allows us to give a formal way to include the electronic susceptibility in the laser equations. This essentially amounts to replace self-consistently in the dynamical equations the frequencies $\omega_{ \pm}$by the instantaneous frequencies $\dot{E}_{ \pm} / E_{ \pm}$.

\section{POLARIZATION SWITCHING IN THE FUNDAMENTAL TRANSVERSE MODE}

\subsection{Dynamical equations}

The dynamical evolution equations of the SFM extended to include the spectral dependence of the material response to the optical field are $^{8}$ : 


$$
\begin{aligned}
& \dot{E}_{ \pm}(t)=-\kappa E_{ \pm}-i \frac{a \Gamma}{2} P_{ \pm}-\left(\gamma_{a}+i \gamma_{p}\right) E_{\mp}+\sqrt{\beta D_{ \pm}} \xi_{ \pm}(t) \\
& \dot{D}_{ \pm}(t)=\mu / 2-A D_{ \pm}-B D_{ \pm}^{2}-\gamma_{j}\left(D_{ \pm}-D_{\mp}\right)-\frac{a}{2 i}\left(P_{ \pm} E_{ \pm}^{*}-c . c .\right) \\
& P_{ \pm}(t) \simeq \varepsilon_{0} \chi\left(\Omega+i \frac{\dot{E}_{ \pm}}{E_{ \pm}}, D_{ \pm}\right) E_{ \pm} .
\end{aligned}
$$

The meaning of the different parameters and typical values used in our numerical simulations are given in Table 1. We have normalized the fields such that $\left|E_{ \pm}\right|^{2}$ is proportional to the photon densities, $n_{e}$ and $n_{g}$ are the reference phase and group refractive indices, $a=\Omega /\left(\varepsilon_{0} n_{g} n_{e}\right)$ is the gain constant, and $\Gamma$ is the confinement factor to the active region. The injected current divided by the carrier density at transparency $\left(N_{t}\right)$ is $\mu=\mathcal{I} /\left(e V N_{t}\right)$ in such a way that at transparency we have $\mu_{t}=A+B$. The current normalized to its transparency value is $J \equiv \mu / \mu_{t}$. The inverse of the carriers life-times is $\gamma_{e} \equiv A+2 B D_{ \pm}$. Spontaneous emission processes, $\xi_{ \pm}(t)$, are modeled by two independent Gaussian white noise sources with properties, $\left\langle\xi_{ \pm}(t)\right\rangle=0$ and $\left\langle\xi_{ \pm}(t) \xi_{ \pm}^{*}\left(t^{\prime}\right)\right\rangle=2 \delta\left(t-t^{\prime}\right)$.

\begin{tabular}{|c|c|c|}
\hline Parameter & Meaning & Value \\
\hline$\varepsilon_{0} a \chi_{0}$ & effective gain constant & $4 \cdot 10^{4} \mathrm{~ns}^{-1}$ \\
\hline$\Gamma$ & confinement factor & 0.045 \\
\hline$\gamma_{\perp}$ & material polarization decay & $10 \mathrm{ps}^{-1}$ \\
\hline$\sigma$ & band-gap shrinkage & 0.2 \\
\hline$b$ & unpumped contribution to $\chi$ & $2 \times 10^{4}$ \\
\hline$\kappa$ & mirror loses & $300 \mathrm{~ns}^{-1}$ \\
\hline$\gamma_{a}$ & linear dichroism & $0.5 \mathrm{~ns}^{-1}$ \\
\hline$\gamma_{p}$ & linear birefringence & $6-30 \mathrm{~ns}^{-1}$ \\
\hline$A$ & non-radiative recombination rate & $1.0 \mathrm{~ns}^{-1}$ \\
\hline$B$ & bimolecular recombination rate & $0.1 \mathrm{~ns}^{-1}$ \\
\hline$\gamma_{j}$ & spin flip rate & $50 \mathrm{~ns}^{-1}$ \\
\hline$\beta$ & spontaneous emission rate & $10^{-5} \mathrm{~ns}^{-1}$ \\
\hline
\end{tabular}

Table 1. Parameters involved in Eqs. (8)-(10), their meaning and typical values.

Eqs. (8)-(10) together with (1) have steady state solutions (for $\beta=0$ ) which correspond to two orthogonal states of linearly polarized (LP) emission depending on whether $E_{+}$and $E_{-}$lock at a phase difference $\psi=0$ or $\pi / 2$. For definiteness, we label them as $\hat{x}$ and $\hat{y}$, respectively, and for $\gamma_{p}>0$ they correspond to the shorter and longer wavelength, respectively. Their frequency splitting is roughly $\Delta \omega=2 \gamma_{p}$. These solutions can be written as,

$$
E_{ \pm}(t)=Q e^{-i \omega_{0} t \pm \psi}, \quad D_{ \pm}=D_{0},
$$

with $\psi=0$ in $\hat{x}$-polarized states and $\psi=\pi / 2$ in $\hat{y}$-polarized states. Inserting eqs. (11) into eq. (8) we obtain with eq. (10) a set of two non-linear algebraic equations for $\left(\omega_{0}, D_{0}\right)$ :

$$
\begin{aligned}
\Gamma \frac{a}{2} \operatorname{Im\chi }\left(\Omega+\omega_{0}, D_{0}\right) & =\kappa \pm \gamma_{a}, \\
\Gamma \frac{a}{2} \operatorname{Re\chi }\left(\Omega+\omega_{0}, D_{0}\right) & =\omega_{0}-\left( \pm \gamma_{p}\right) .
\end{aligned}
$$

From eq. (9) we obtain the total optical power proportional to

$$
2 Q^{2}=\frac{\Gamma \mu_{t}}{2}\left[\frac{J-J_{t h}}{\kappa \pm \gamma_{a}}\right],
$$

where the sign $+(-)$ corresponds to $\hat{x}$-polarized ( $\hat{y}$-polarized) solution. The threshold current is given by $J_{t h}=$ $2\left(A D_{0}+B D_{0}^{2}\right) / \mu_{t}$ which depends on temperature through the value of $\Delta$. 


\subsection{Non-thermal and thermal switching}

\subsubsection{Stability diagrams}

The linear stability analysis of the linearly polarized solutions allows to determine the domain in parameter space where each LP state is stable and thus the possible occurrence of polarization switching. PS can occur as the system is brought from one parameter region to another region of different stability properties.

Typical stability results for a relatively small value of the birefringence $\left(\gamma_{p} / A=6\right)$ are shown in Fig. 2(a) in the $J-\Delta$ plane. There is a minimum threshold for $\Delta=\Delta_{m}$ corresponding to the cavity resonance being aligned with the gain peak. Generally, for a given $\Delta$, the LP state selected at threshold is the closest to the gain peak, i. e., $\hat{x}$ for $\Delta<\Delta_{m}$ and $\hat{y}$ for $\Delta>\Delta_{m}$. This is not true when a positive dichroism $\left(\gamma_{a} / A=0.5\right)$ is introduced favoring the $\hat{y}$ component. Above the threshold line we find a region (III) in which only $\hat{y}$ is stable and a region (I) where only $\hat{x}$ is stable. A bistability region (II) also appears, extending from below to above $\Delta_{m}$, due to nonlinear gain saturation induced by the lasing mode. PS occurs as the system is brought from III to I or viceversa. Finally, a region IV may appear where neither of the linearly polarized states is stable as was already described in ref. [4].

Fig. 2(b) displays a typical stability diagram for a higher birefringence $\left(\gamma_{p} / A=30\right)$. An important difference with Fig. 2(a) is that now, $\hat{x}$ is stable on almost the whole red side of the gain peak for currents near threshold because it is favored by a negative dichroism. Therefore, no PS at constant temperature appears for detunings $\Delta<\Delta_{m}$. Instead, a PS occurs for constant $J$ as $\Delta$ is varied across $\Delta_{m}$. Within our framework, we call this PS "thermally induced" since it appears by scanning $\Delta$, either by changing substrate temperature or by device self-heating as the current is increased (see arrow in Fig. 2(b)).

Therefore we find two possible, independent ways to produce a PS. One that typically occurs for relatively large birefringence (2(b)) is "thermally induced" and corresponds to the mechanism discussed by Choquette et al.. ${ }^{13}$ A second mechanism can typically occur for small birefringences keeping constant $\Delta$ while increasing $J$ (see the arrows in Fig. 2(a)). We call this second mechanism "non-thermal" since it occurs at constant $\Delta$ and while staying on one of the sides of the gain peak. It is associated with the polarization switching mechanisms discussed in ref. [4]. It should be noted that in this case, the switching takes place from the LP state with the longer wavelength to that with shorter wavelength. For example in the path of arrow (2) in 2(a) the mode selected at threshold is the one with largest unsaturated material gain, but increasing current it switches to the one of smaller unsaturated gain which is also unfavorable by the sign of the linear dichroism.
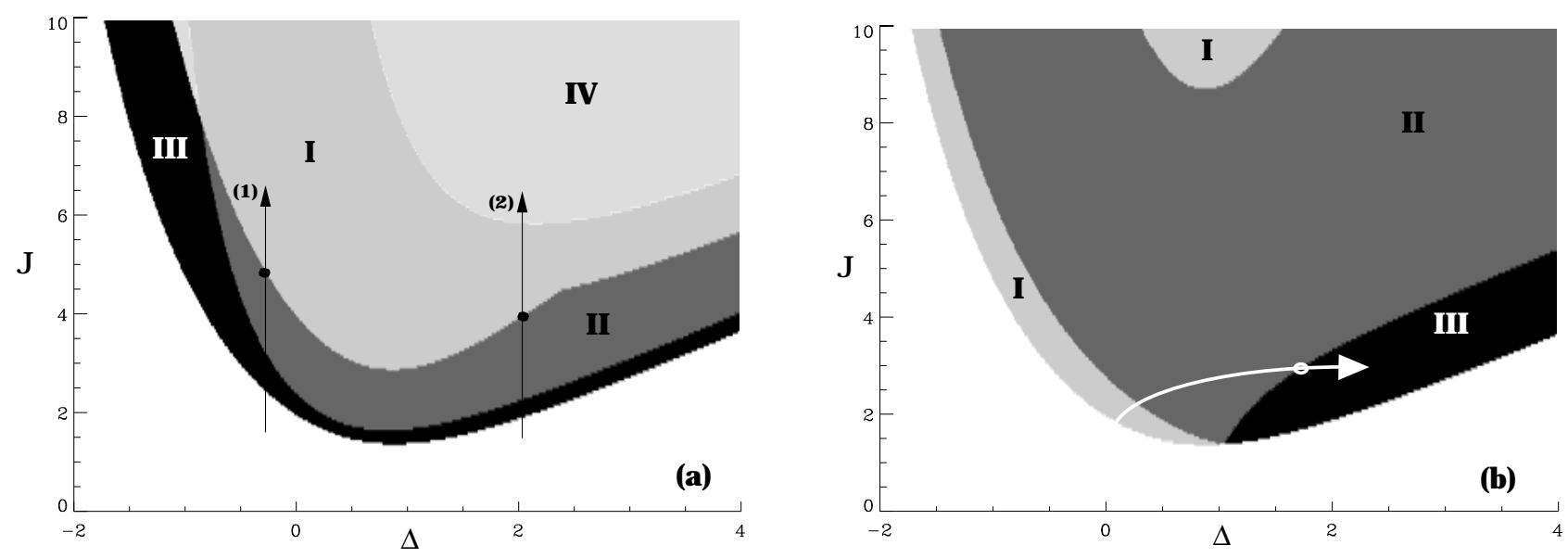

Figure 2. Current-detuning linear stability diagram for the linearly polarized solutions. The lower curves gives the dependence of threshold current on the detuning $\Delta$. Parameters used are those in Table 1 with (a) $\gamma_{p} / A=6$, $\gamma_{a} / A=0.5$ and (b) $\gamma_{p} / A=30, \gamma_{a} / A=-0.5$. The stability regions are identified as follows: I only $\hat{x}$ stable, II $\hat{x}$ and $\hat{y}$ stable, III only $\hat{y}$ stable, IV $\hat{x}$ and $\hat{y}$ unstable. 


\subsubsection{Current scanning at constant temperature: Non-thermally induced PS}

Fig. 3 shows examples of dynamical evolution through a PS obtained from the numerical integration of the equations (8)-(10) in the case of low birefringece $\left(\gamma_{p} / A=6\right)$. We plot the time evolution of the intensities $I_{x}$ and $I_{y}$ of the linearly polarized field components as the injected current, $J$, is raised by steps from $0.85 J_{t h}$ up to $4 J_{t h}$. Results are shown for two values of the detuning parameter: (a) $\Delta=-0.25$, and (b) $\Delta=2$. These two values correspond to the two arrows indicated in Fig. 2(a). In both cases a PS $(\hat{y} \rightarrow \hat{x})$ occurs, but the dynamical evolution is qualitatively different. In (a) the switching is smooth, i.e. the $\hat{y}$-linearly polarized solution becomes unstable when the current is increased, and the switching occurs through intermediate states with complicated dynamics. This complex dynamics can be attributed to the competition between the two polarization states: in this case dichroism can compensate the difference of modal gain for $\Delta<\Delta_{m}$ yielding two polarization components with nearly the same effective gain. On the other hand, in $3(\mathrm{~b})$ there is a sharp PS between the two linearly polarized states.

(a)

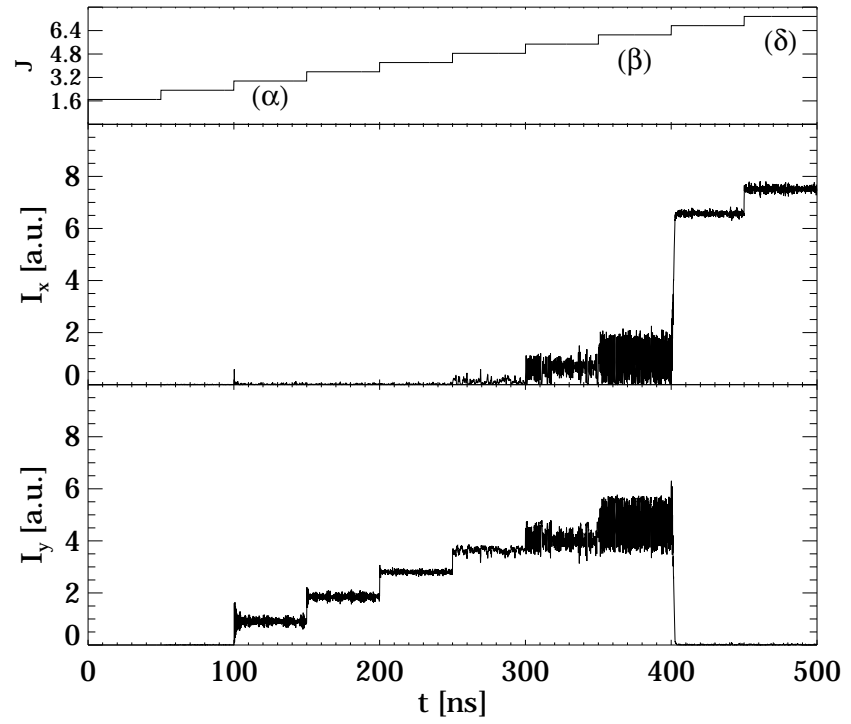

(b)

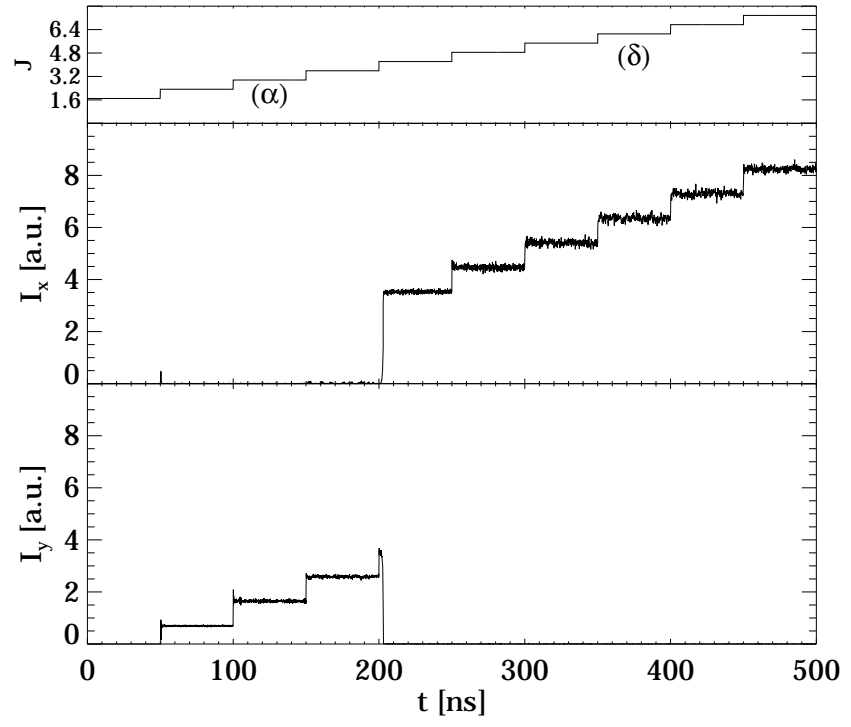

Figure 3. Non-thermal switching obtained increasing the injection current along the paths indicated in Fig. 2(a). (a) $\Delta=-0.25$, and (b) $\Delta=2$. ( $\alpha$ ) corresponds to a $\hat{y}$-linearly polarized state, $(\delta)$ to a $\hat{x}$-linearly polarized, and $(\beta)$ is an intermediate dynamical state.

\subsubsection{Current scanning with temperature variations: Thermally induced PS}

When the injection current is slowly increased, the temperature of the device also increases due to device self-heating. The consequence is that an effective diagonal path in the $J-\Delta$ stability diagram is followed (see fig. 2(b)). We assume a simple dependence of the detuning on the current given by,

$$
\dot{\Delta}=\frac{\left(\Delta-\Delta_{0}\right)-a\left(J-J_{0}\right)}{\tau_{t}},
$$

with $\tau_{t}$ the thermal time, and where $\Delta_{0}, J_{0}$ are initial conditions. The parameter $a$ is related to the thermal resistance of the device. ${ }^{14}$ We take $a=2$. A typical time scale for the thermal response time is $\tau_{t}=1 \mu \mathrm{s}$. The situations considered in the previous subsection with PS occurring for constant $\Delta$ can be experimentally accessed by applying injection current pulses of duration smaller than the thermal response time. ${ }^{5}$

In Fig. 4 we show the time evolution of the intensities $I_{x}$ and $I_{y}$ of the linearly polarized field components when the detuning changes according to eq. (15). The parameters chosen are those of Fig. 2(b). The device is prepared with an initial negative detuning $\Delta_{0}<\Delta_{m}$. A switching $(\hat{x} \rightarrow \hat{y})$ is seen to occur with a thermal roll-over in the polarization components due to the change of the threshold current with temperature. The point of PS is the one predicted by the linear analysis shown in Fig. 2(b). 


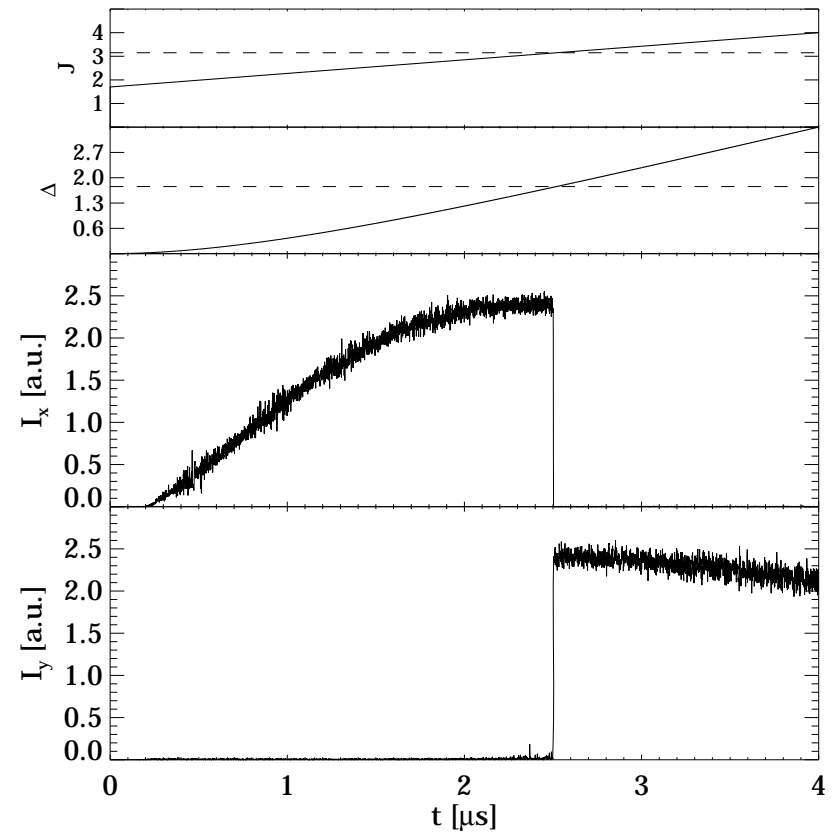

Figure 4. Thermally induced switching. The detuning increases according to eq. (15). Parameters of Fig. 2(b).

\subsection{Polarization resolved power spectrum}

Generally speaking the power spectrum gives important information of the device operation. ${ }^{15}$ More specifically, polarization resolved power spectra yield information on polarization partition noise which might cause a degradation of the signal-to-noise ratio. The properties of these spectra and their change through a PS also give interesting information on PS mechanisms. We present here results for power spectra corresponding to the PS of Fig. 3(a).

Let $I_{u}, I_{v}$ be the intensity of each orthogonal polarization component $(u=+, v=-$ in the circular basis or $u=x, v=y$ in the linear basis). The power spectrum of each component is defined by:

$$
S_{u, v}(\omega)=<\left|\delta \tilde{I}_{u, v}(\omega)\right|^{2}>,
$$

and the power spectrum for the total intensity is:

$$
S_{I}(\omega)=<\left|\delta \tilde{I}_{u}(\omega)+\delta \tilde{I}_{v}(\omega)\right|^{2}>,
$$

where $\langle\cdot\rangle$ means the average over different realizations of the noise.

In Fig. 5, we show the power spectra corresponding to the states labeled as $(\alpha),(\beta),(\delta)$ in 3(a). State $\alpha$ is a $\hat{y}$-polarized state far below the polarization switching. From panels $5(\mathrm{a})$ and $5(\mathrm{~b})$ we observe that different useful information can be extracted from the two orthogonal polarization basis that we consider. The $(+/-)$ basis is the natural physical basis for the ideal case of an isotropic cavity. In this basis a peak associated with the birefringence appears in the power spectrum of each component, since birefringence gives a linear coupling of the two circularly polarized components. This peak is missing in the $(\mathrm{x} / \mathrm{y})$ basis. Moreover, intensity fluctuations of the two polarization components in the (+/-) basis are highly anticorrelated at low frequencies, while this anticorrelation is less apparent in the $(\mathrm{x} / \mathrm{y})$ basis. Anticorrelations disappear in both basis for frequencies close to the relaxation oscillation (RO) frequency where a positive correlation between the two components is maximum.

Figs. $5(\mathrm{e})$ and $5(\mathrm{f})$ (state $\delta$ of Fig. 3(a)) correspond to a $\hat{x}$-polarized state far above the polarization switching. The general features are similar to those of the state $\alpha$ described above, except that the anticorrelations observed at low frequency are now strongly reduced. On the contrary the positive correlation at the peak of the RO frequency is maintained. The RO peak occurs at higher frequencies due to the larger injection current. 
$(\alpha)$

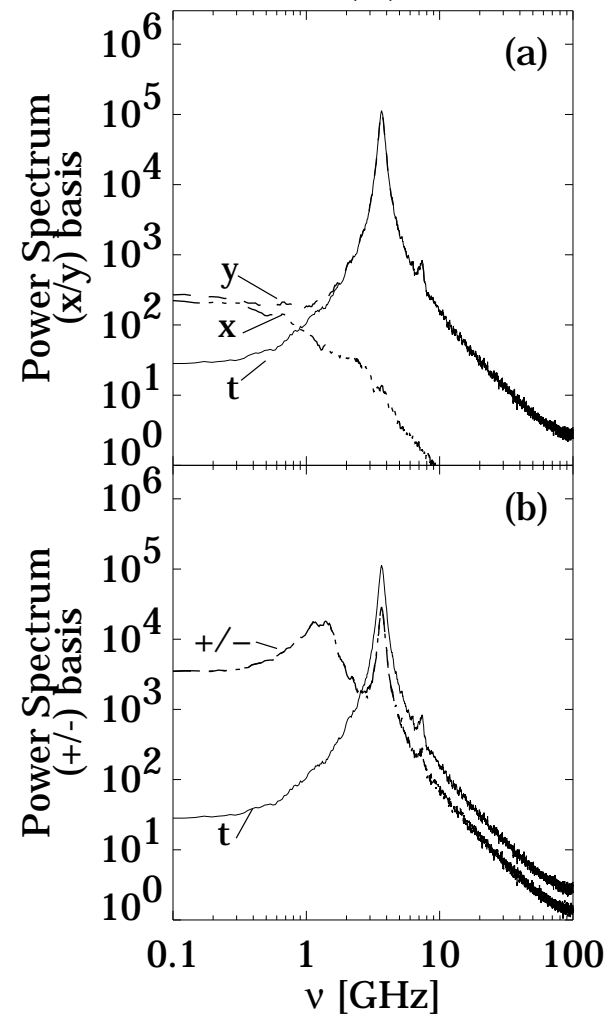

$(\beta)$

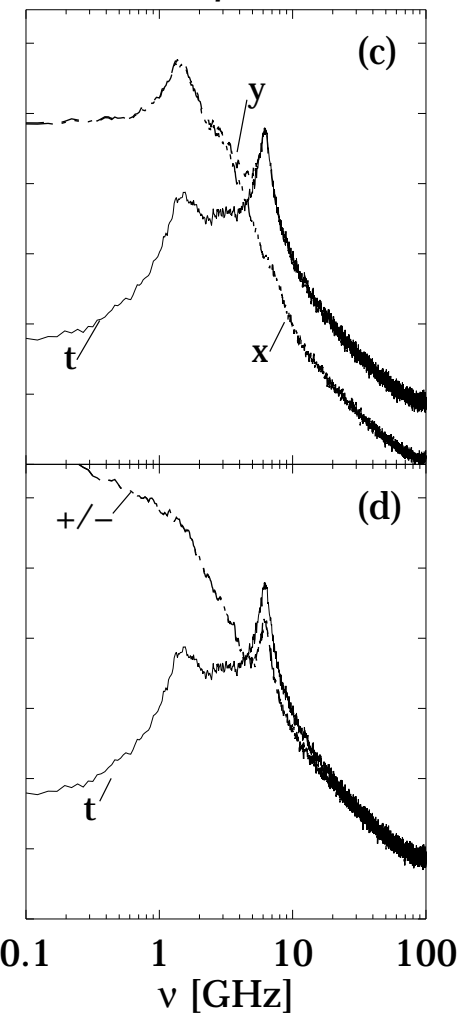

$(\delta)$

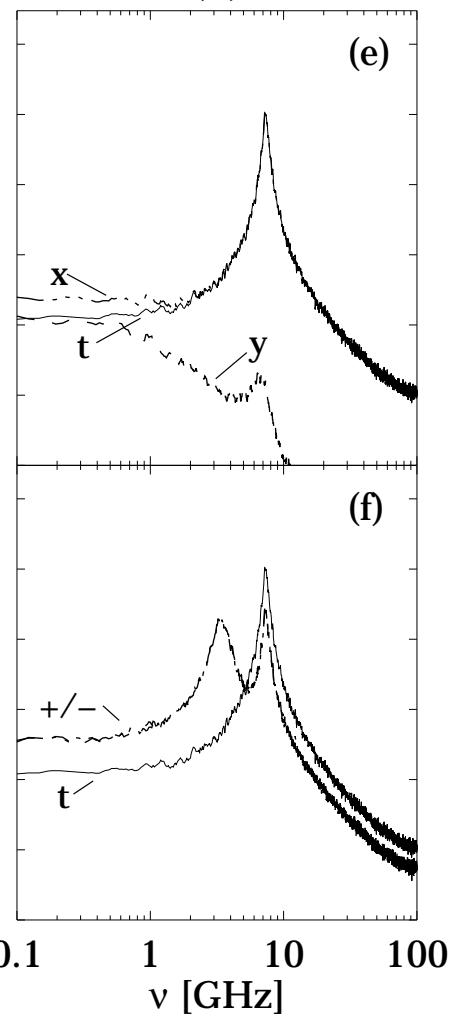

Figure 5. Power spectrum in the linear and circular basis corresponding to the states $\alpha, \beta, \delta$ in Fig. 3(a). The symbols $t, x, y, \pm$ refer to $S_{I}(\omega), S_{x}(\omega), S_{y}(\omega)$, and $S_{ \pm}(\omega)$ respectively.

The $\beta$ state, Figs. 5(c) and 5(d), displays more complex spectra since it is associated with a state of complicated sustained polarization dynamics. The intensity of both polarization components are oscillating in time. This kind of dynamical states appear close to the polarization switching. The corresponding power spectra exhibit peaks at different frequencies associated with the irregular time evolution. A very large anticorrelation appears at low frequency due to polarization partition noise. The peak at the RO frequency with a maximum positive correlation is maintained.

\section{TRANSVERSE DYNAMICS IN GAIN-GUIDED VCSELS}

Our analysis so far implicitly uses as dynamical variables the amplitudes of the different polarizations of a fundamental transverse mode. For gain guided VCSELs the mode profile is not precisely defined and spatial transverse effects might be important. Inclusion of these effects is also necessary to study the emergence of transverse modes of higher order. A generalization of the SFM taking into account both a frequency-dependent QW semiconductor complex susceptibility and the spatial dependence on the region of the active plane is given by

$$
\begin{aligned}
\partial_{t} E_{ \pm}(x, y ; t) & =-\kappa E_{ \pm}-i \frac{a \Gamma}{2} P_{ \pm}+i \frac{c^{2}}{2 n_{e} n_{g} \Omega} \nabla_{\perp}^{2} E_{ \pm}-\left(\gamma_{a}+i \gamma_{p}\right) E_{\mp}+\sqrt{\beta D_{ \pm}} \xi_{ \pm}(x, y ; t) \\
\partial_{t} D_{ \pm}(x, y ; t) & =\mathcal{J}(x, y) \mu(t) / 2-A D_{ \pm}-B D_{ \pm}^{2}-\gamma_{j}\left(D_{ \pm}-D_{\mp}\right)+\mathcal{D} \nabla_{\perp}^{2} D_{ \pm}-\frac{a}{2 i}\left(P_{ \pm} E_{ \pm}^{*}-c . c .\right) \\
P_{ \pm}(x, y ; t) & \simeq \varepsilon_{0} \chi\left(\Omega+i \frac{\partial_{t} E_{ \pm}}{E_{ \pm}}, D_{ \pm}\right) E_{ \pm} .
\end{aligned}
$$


$E_{ \pm}, D_{ \pm}$, and $P_{ \pm}$have the same meaning as in section 3.1. Diffraction of the optical field is modeled by the transverse laplacian term in eq. (18). The laplacian term in eq. (19) models the carrier diffusion with constant $\mathcal{D}$. The current profile, $\mathcal{J}(x, y)$, together with the carrier diffusion determines the shape of the carrier distribution below threshold. A realistic shape for the current profile for relatively small VCSELs is a supergaussian $\mathcal{J}(r)=$ $\exp \left[-(2 r / \phi)^{6}\right]$, where $\phi$ the active region diameter. $\mu(t)$ allows to apply current steps or ramps. The rest of parameters were previously defined in Table 1 . The numerical integration of eqs. (18)-(20) is delicate and time consuming. We use FFT routines to calculate the diffraction and diffusion terms and an Euler method with noise to update the dynamical variables.

In Fig. 6, we show an example of a numerical integration of eqs. (18)-(20) for a laser of diameter $\phi=12.5 \mu \mathrm{m}$. The laser operates at the positive detuning side $\left(\Delta>\Delta_{m}\right)$ and the $\hat{y}$-solution is selected at threshold. We first show how raising the current from below to above threshold, at $t=0$, the laser switches-on in the $\hat{y}$-polarized solution. At time $t=10 \mathrm{~ns}$ the injection current is increased by a step and the $\hat{y}$-polarized solution switches to the $\hat{x}$-polarized solution. The near field images demonstrate that the overall process takes place in the fundamental transverse mode. Although spatial hole burning can modify the linear stability analysis, the main qualitative features remain unchanged with respect the results presented before when neglecting spatial transverse effects. We are presently using the integration of eqs. (18)-(20) to analyze the emergence of higher order transverse modes and the correlations between polarization and transverse mode selection.

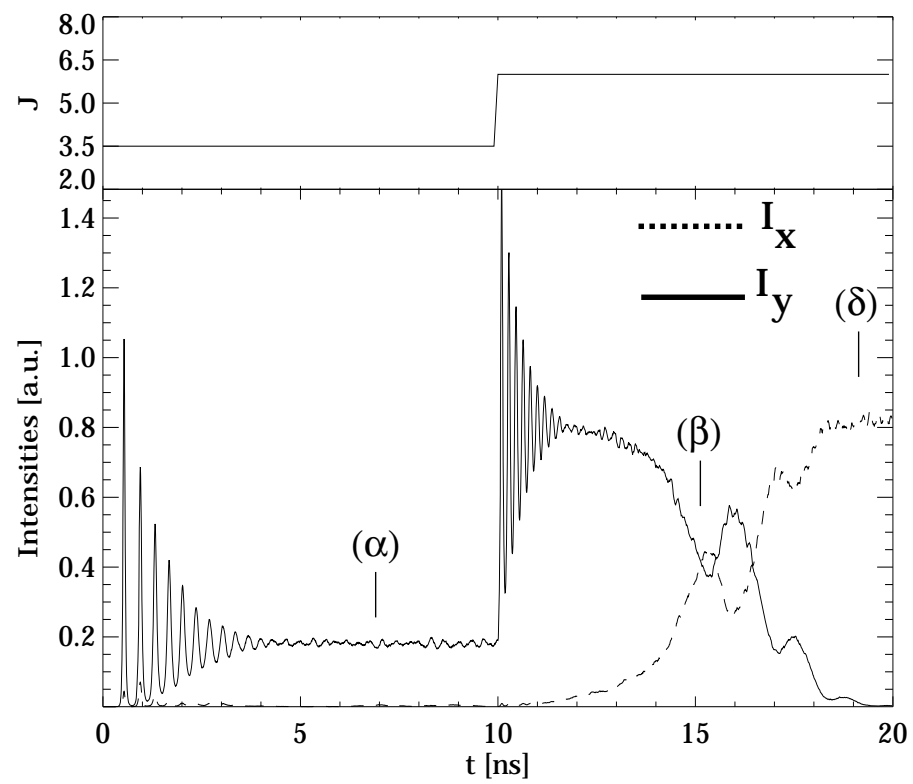

$(\alpha)$
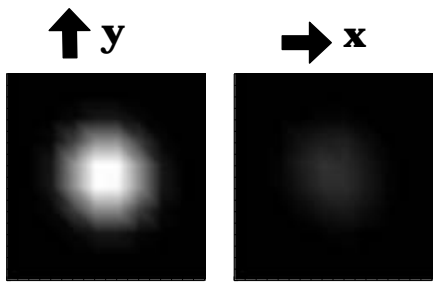

$(\beta)$
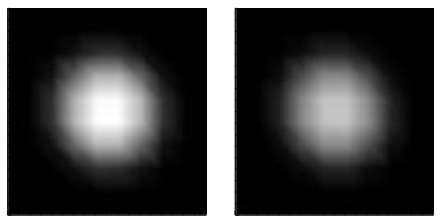

$(\delta)$
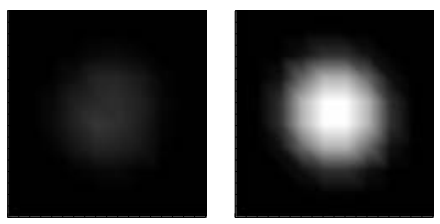

Figure 6. A PS in the fundamental transverse mode. Near field images show the transverse beam profile during the PS. The parameters used are $\Delta=1, \gamma_{a}=0, \gamma_{p} / A=1, \gamma_{j} / A=150$ and $a / A=1.3 \times 10^{4}$.

\section{ACKNOWLEDGMENTS}

Work partially supported by the European Commission (TMR Project FMRX-CT96-0066 and IHP-RTN1-199900279) and by DGICYT and CICYT (Spain) Projects PB94-1167 and TIC99-0645. 


\section{REFERENCES}

1. M. San Miguel, "Polarization Properties of VCSELs" in Semiconductor Quantum Optoelectronics, Ed. A. Miller, Institute of Physics (1999), and references therein.

2. A. K. Jansen van Doorn, M. P. van Exter and J. P. Woerdman, "Elasto-optic anisotropy and polarization orientation of VCSELs", Appl. Phys. Lett. 69, 1041-1043 (1996); M. P. van Exter, R. F. M. Hendriks, and J. P. Woerdman, "Physical insight into the polarization dynamics of semiconductor vertical-cavity lasers" Phys. Rev. A 57, 2080-2090, (1998).

3. M. San Miguel, Q. Feng, J.V. Moloney, "Light-polarization dynamics in surface-emitting semiconductor lasers", Phys. Rev. A 52, 1728-1739, (1995).

4. J. Martín-Regalado, F. Prati, M. San Miguel, and N.B. Abraham, "Polarization properties of vertical-cavity surface-emitting lasers", IEEE J. Quantum Electron. 33, 765-783,(1997).

5. J. Martín-Regalado, J. L. A. Chilla, J. J. Rocca and P. Brusenbach, "Polarization switching in vertical-cavity surface-emitting lasers observed at constant active region temperature", Appl. Phys. Lett. 70, 3350-3352, (1997).

6. K. D. Choquette, R. P. Schneider Jr., K. L. Lear and R. E. Leibenguth, "Gain-Dependent Polarization Properties of Vertical-Cavity Lasers", IEEE J. Select. Topics Quantum Electron. 1, 661-666, (1995).

7. J. Martín-Regalado, S. Balle and M. San Miguel, "Polarization and transverse-mode dynamics of gain-guided vertical-cavity surface-emitting lasers", Opt. Lett. 22, 460-462, (1997).

8. S. Balle, E. Tolkachova, M. San Miguel, J.R. Tredicce, J. Martín-Regalado and A. Gahl, "Mechanisms of polarization switching in single-transverse-mode VCSELs: Thermal shift and nonlinear semiconductor dynamics", Opt. Lett. 24, 1121-1123, (1999).

9. R.S. Britton, T. Grevatt, A. Malinowski, R.T. Harley, P. Perozzo, A.R. Cameron and A. Miller, "Room temperature spin relaxation in GaAs/AlGaAs multiple quantum wells", Appl. Phys. Lett. 73, 2140-2142, (1998).

10. S. Balle, "Simple analytical approximations for the gain and refractive index spectra in quantum-well lasers", Phys. Rev. A 57, 1304-1312, (1998).

11. S. Balle and A. Gahl, "Influence of the confinement factor on the wavelength-dependent output properties of a tapered traveling wave semiconductor amplifier", IEEE Phot. Tech. Lett. 11, 1375-1377, (1999).

12. T. Rössler, R.A. Indik, G.K. Harkness, and J.V. Moloney, "Modeling the interplay of thermal effects and transverse mode behaviour in native-oxide-confined vertical cavity surface-emitting lasers", Phys. Rev. A 58, 3279-3292, (1998).

13. K.D. Choquette, D.A. Richie, and R.E. Leibenguth, "Temperature dependence of gain-guided vertical-cavity surface emitting laser polarization", Appl. Phys. Lett. 64, 2062-2064, (1994).

14. W. Nakwaski and M. Osinski, "Thermal properties of vertical-cavity surface-emitting semiconductor lasers", Progress in Optics XXXVIII, 165-262, (1998).

15. K. Ebeling in Semiconductor Quantum Optoelectronics, Ed. A. Miller, Institute of Physics (1999). 